\title{
PHENOLIC CONTENT, ANTIOXIDANT ACTIVITY AND PHARMACEUTICAL AVAILABILITY OF HYDROGELS WITH EXTRACTS OF ROSMARINUS OFFICINALIS L. AND SAMBUCUS NIGRA L.
}

\author{
MONIKA MICHALAK ${ }^{1 *}$, AGNIESZKA ZIELIŃSKA², and KATARZYNA PARADOWSKA²
}

'Department of Dermatology, Cosmetology and Aesthetic Surgery, Institute of Medical Sciences, Collegium Medicum, Jan Kochanowski University, IX Wieków Kielce 19a, 25-317 Kielce, Poland

${ }^{2}$ Chair of Physical Pharmacy and Bioanalysis, Department of Physical Chemistry, Faculty of Pharmacy, Medical University of Warsaw,

Banacha 1, 02-097 Warsaw, Poland

\begin{abstract}
New active ingredients, including those of plant origin, are still sought in the pharmaceutical and cosmetics industry. The present study was focused on the determination of the polyphenolic content and the confirmation of antioxidant properties of rosemary and elderflower extracts. Investigations were also carried out to define the pharmaceutical availability of hydrogels with the tested extracts. This study has demonstrated that the extract of rosemary herb is characterized by greater DPPH scavenging activity. It also contains a higher content of polyphenols, including flavonoids. The research of diffusion capacity of plant extracts from hydrogels showed that the extract from rosemary herb is more efficiently released. Extracts of Rosmarinus officinalis L. and Sambucus nigra L. can be used as natural products with high antioxidant properties.
\end{abstract}

Keywords: antioxidant, pharmaceutical availability, plant extract, polyphenols, Rosmarinus officinalis, Sambucus nigra

Plant extracts, due to the versatility of biologically active compounds (including polyphenols, essential oils, vitamins), are often used in cosmetic products, natural drugs, as well as food supplements. They are components with a multifunctional effect, including antioxidant one (1). The antioxidant potential is directly related to the radical scavenging ability. High levels of radicals can cause far-reaching damage to cellular structures. Also, the skin, although it has natural defense mechanisms against the action of radicals, in the case of their excessive production is exposed to the effects of the so-called oxidative stress (2). Radicals attack the ceramides of intercellular cement and destroy lipid components of sebum, which contribute to the formation of products irritating the skin and causing inflammatory and allergic reactions. The effect of oxidative stress is the accelerated wrinkle formation, loss of elasticity and dryness of the skin, uneven pigmentation and discoloration, susceptibility to irritation, inflammatory and allergic reactions of the skin, and slowing down the process of wound healing $(3,4)$.
Antioxidants play an important role in protecting the skin against oxidative damage $(4,5)$. Polyphenols, including flavonoids, belong to a group of natural compounds characterized by strong antioxidant properties and supporting the defense and regenerative mechanisms of the skin $(6,7)$. These compounds have a protective effect on ascorbic acid, which is why they have a beneficial impact on collagen biosynthesis. Polyphenols also soothe skin irritations and redness, accelerate the natural regeneration of the epidermis, improve microcirculation, and protect against harmful external factors, including UV radiation $(8,9)$.

An important source of biologically active compounds, including those showing antioxidant effects, is rosemary and elderflower $(10,11)$. Rosemary (Rosmarinus officinalis L.) is an evergreen shrub from the family of Lamiaceae. Medicinal and cosmetic/fragrance raw material is a rosemary leaf, containing flavonoids (such as luteolin, genkwanin, hesperidin, isorhamnetin, diosmin), phenolic acids

* Corresponding author: e-mail: monika.michalak@ujk.edu.pl 
(mainly rosmarinic acid, as well as caffeic, chlorogenic, and ferulic acid), diterpenoids (mainly rosmanol, carnosol, carnosic acid, epirosmanol), triterpenoids (betulinic, ursolic, or oleanolic acid), alkaloids, phytosterols, tannins, saponins and essential oil (10, 12-14). Rosemary leaf is known as an analgesic, stimulating vitality, antiseptic and aphrodisiac agent. It improves blood circulation and is used internally in digestive disorders and contractions of the gastrointestinal tract $(13,14)$. Rosemary is used in skin and hair care, owing to its strong antioxidant, anti-inflammatory, and antibacterial properties (15). It is a component of anti-wrinkle and firming preparations (14). Sambucus nigra L., also called black elder, is a shrub from the family Caprifoliaceae. The elderflowers contain flavonoids (quercetin, isoquercetin, kaempferol, myricetin, rutin, nicotiflorine) as well as phenolic acids (caffeic, chlorogenic, p-coumaric, ferulic, gallic, and syringic acid) and triterpene ( $\alpha$ - and $\beta$-amarine, ursolic, oleanolic acid). Organic acids and essential oils are also present $(11,16)$. S. nig$r a$ is known as a diaphoretic, antipyretic, immunomodulatory, antibacterial, antifungal, expectorant, and diuretic medicinal product (11). Both the berries and the flowers of the elder plant are used as dietary supplements for minor diseases such as colds, often served as a tea, or in a capsule, also in topical applications due to its antioxidant, astringent, and anti-inflammatory properties. The black elder extract was found to be an effective component of preparations protecting against UVA and UVB radiation (14). Sambuci flos is recommended for mature, tired, and colorless skin (17).

Despite the abundance of information regarding the significance and application possibilities of $R$. officinalis L. and S. nigra L. in the pharmaceutical and cosmetics industry, there is still insufficient data regarding their use in preparations applied topically on the skin.

An important issue for the pharmaceutical and cosmetics industry, which determines the effectiveness of the active substance, is its penetration into the skin. The assessment of permeability, i.e. the route through individual layers of the skin, up to the general circulation, except for substances used therapeutically in dermatology, may also apply to some substances used in cosmetology. The penetration of active substances deep into the skin is determined by factors such as the condition of the skin, the physicochemical nature of the compound, and the type of the base. Penetration can be accelerated by using substances that provide relaxation of stratum corneum cells, including alcohols or glycols. A modern method determining the availability of active ingredients contained in a pharmaceutical or cosmetic product is the study of release kinetics from formulations, such as emulsions or gels (18). This experiment allows the assessment of the ability of the active substance to diffuse from the substrate through the membrane into a suitable solution. The results of the study on the availability of the active substance are influenced by such factors as proper selection of the membrane, acceptor liquid, and process temperature. To best reflect the physiological conditions, the specific conditions under which the test is performed should be preserved. The membrane imitating the skin barrier should be characterized by high porosity and low thickness. The membrane should also be indifferent to the acceptor fluid and the formulation medium. The main function of the membrane is to keep the test preparation in a solid place and to prevent the release of particles of excipients into the acceptor fluid. Another important parameter affecting the results of the release test is the $\mathrm{pH}$ of the acceptor fluid which should be close to the $\mathrm{pH}$ of the human skin ( $\mathrm{pH}$ range 5-6). The substance that we want to examine must be soluble in the acceptor fluid. In the case of substances that are soluble in water, the phosphate buffer is most often used as a medium, whereas when the active substance is not dissolved in water, an aqueous-alcoholic solution is used. The test is usually carried out at 25,32 or $37^{\circ} \mathrm{C}$, while the time of analysis depends on the solubility of the active substance being tested (19).

The aim of the present research was to characterize the chemical profile of glycolic extracts of rosemary and elderflower, their antioxidant activities, and to assess the diffusion capacity of plant extracts from self-prepared hydrogels.

\section{EXPERIMENTAL}

\section{Plant materials and chemicals}

Glycolic extract of rosemary 5\% (Naturex, Poland) and glycolic extract of elderflower 5\% (Naturex, Poland) were used in the study. Reagents used to make hydrogels were ethanol $96 \%$ p.a. (Avantor Performance Materials, Poland), methanol p.a. (Chempur, Poland), acetic acid $80 \%$ p.a. (Chempur, Poland), Carbopol ${ }^{\circledR}$ Ultrez 10 Polymer (Lubrizol, USA), 1.2-propanodiol p.a. (Avantor Performance Materials, Poland), triethanolamine p.a. compatible with European Pharmacopoeia VI (Chempur, Poland), deionized water. All standards, reagents, and solvents, used for the HPLC analysis, were of analytical or gradient grade. HPLC standards: rosmarinic acid (CAS 20283-92-5), rutin hydrate (CAS 207671-50-9), quercetin (CAS 117-39-5) were purchased from Sigma-Aldrich, with purity $\geq 99 \%$. 


\section{Determination of the total polyphenols content}

Total polyphenols content (TPC) of glycolic extract of rosemary and glycolic extract of elderflower was determined using the Folin-Ciocalteu reagent (20), with gallic acid as the reference standard. A standard curve of gallic acid was prepared using acid solutions from 50 to $500 \mathrm{mg} / \mathrm{L}$ (standard curve equation: $\mathrm{Abs}=0.0011 \mathrm{x}-0.0016\left(\mathrm{R}^{2}=0.9996\right)$, where Abs - absorbance at $325 \mathrm{~nm}, \mathrm{x}$ - concentration of gallic acid $(\mathrm{mg} / \mathrm{L})$. The results were expressed as gallic acid equivalent (mg GAE per $100 \mathrm{~mL}$ of extract). Every sample was prepared in triplicate. TPC was determined spectrophotometrically using Evolution 60S UV-Vis spectrophotometer (Thermo Scientific, USA).

\section{Determination of the total flavonoids content}

Total flavonoids content (TFC) of glycolic extract of rosemary and glycolic extract of elderflower were measured according to the method described by Kim et al. (21), using catechin as a standard. A standard curve of catechin was prepared using acid solutions from 0.05 and $0.40 \mathrm{mg} / \mathrm{mL}$ (standard curve equation: $\mathrm{Abs}=1.4083 \mathrm{x}-0.003\left(\mathrm{R}^{2}=0.9867\right)$, where Abs - absorbance at $510 \mathrm{~nm}, \mathrm{x}$ - concentration of catechin $(\mathrm{mg} / \mathrm{mL})$. The results were expressed as catechin equivalent (mg CE per $100 \mathrm{~mL}$ of extract). Every sample was prepared in triplicate. TFC was determined spectrophotometrically using Evolution 60S UV-Vis spectrophotometer (Thermo Scientific, USA).

\section{HPLC analysis}

The HPLC-RI quantitative analysis of extracts was carried out using a Hitachi Chromaster HPLC system with DAD detector and a Purospher STAR $\mathrm{RP}-18(5 \mu \mathrm{m}, 4 \times 250 \mathrm{~mm})$ column at $30^{\circ} \mathrm{C}$.

Determination of rosmarinic acid in rosemary glycolic extract: mobile phase: (A) $0.1 \%$ formic acid in $\mathrm{H}_{2} \mathrm{O}(\mathrm{v} / \mathrm{v})$. (B) $0.1 \%$ formic acid in acetonitrile $(\mathrm{v} / \mathrm{v})$, flow rate: $1 \mathrm{~mL} / \mathrm{min}$. The separation conditions: a linear gradient $10-40 \% \mathrm{~B}$ in $0-25 \mathrm{~min}$ followed by the isocratic elution with $10 \%$ of B (25$35 \mathrm{~min}$ ) to equilibrate the column. The chromatograms were monitored at $330 \mathrm{~nm}$. The concentration of rosmarinic acid was determined using a calibration curve (Table 1). Figure la shows chromatograms of rosemary extract and rosmarinic acid standard (RT $20.2 \mathrm{~min})$.

Determination of rutin and quercetin in elderflower glycolic extract: mobile phase: (A) $0.1 \%$ formic acid in $\mathrm{H}_{2} \mathrm{O}(\mathrm{v} / \mathrm{v})$. (B) $0.1 \%$ formic acid in acetonitrile $(\mathrm{v} / \mathrm{v})$, flow rate: $1 \mathrm{~mL} / \mathrm{min}$. The separation conditions: a linear gradient $10-20 \% \mathrm{~B}$ in $0-15 \mathrm{~min}, 20-90 \% \mathrm{~B}$ in $15-40 \mathrm{~min}$, then the isocratic elution with $10 \%$ of $\mathrm{B}(40-50 \mathrm{~min})$ to equilibrate the column. The chromatograms were monitored at $330 \mathrm{~nm}$. The concentration of rutin was determined using a calibration curve (Table 1). Figure $1 \mathrm{~b}$ shows chromatograms of elderflower extract standard and spiked with rutin and quercetin (rutin RT $20.5 \mathrm{~min}$, quercetin RT $28.5 \mathrm{~min}$ ).

The linearity of the methods was assessed based on the value of the coefficient of determination $\left(\mathrm{R}^{2}\right)$ of the calibration curve. The limit of detection (LOD) and limit of quantification (LOQ) concentrations were set using equations: $\mathrm{LOD}=3.3 \times \sigma / \mathrm{S}$ and $\mathrm{LOQ}=10 \times \sigma / \mathrm{S}$, where $\sigma$ is the standard deviation of the $\mathrm{y}$-intercept and $\mathrm{S}$ is the slope of the calibration curve. The results of method accuracy analysis

Table 1. Calibration curves, concentration range, limit of detection, and limit of quantification for standards.

\begin{tabular}{|c|c|c|c|c|c|}
\hline Compound & Calibration curve $^{\mathrm{a}}$ & $\mathrm{R}^{2}$ & Linear range $(\mathrm{mg} / \mathrm{mL})$ & $\mathrm{LOD}(\mathrm{mg} / \mathrm{mL})$ & $\mathrm{LOQ}(\mathrm{mg} / \mathrm{mL})$ \\
\hline Rosmarinic acid & $\mathrm{A}=27481709 \mathrm{c}-288575$ & 1.000 & $0.050-0.400$ & 0.020 & 0.070 \\
\hline Rutin & $\mathrm{A}=14410746 \mathrm{c}-134479$ & 1.000 & $0.050-0.400$ & 0.010 & 0.030 \\
\hline
\end{tabular}

${ }^{\mathrm{a}} \mathrm{A}$ - peak area $\left(\mu \mathrm{V}^{*} \mathrm{sec}\right), \mathrm{c}-$ concentration $(\mathrm{mg} / \mathrm{mL})$ of the compound, LOD - limit of detection, $\mathrm{LOQ}$ - limit of quantification
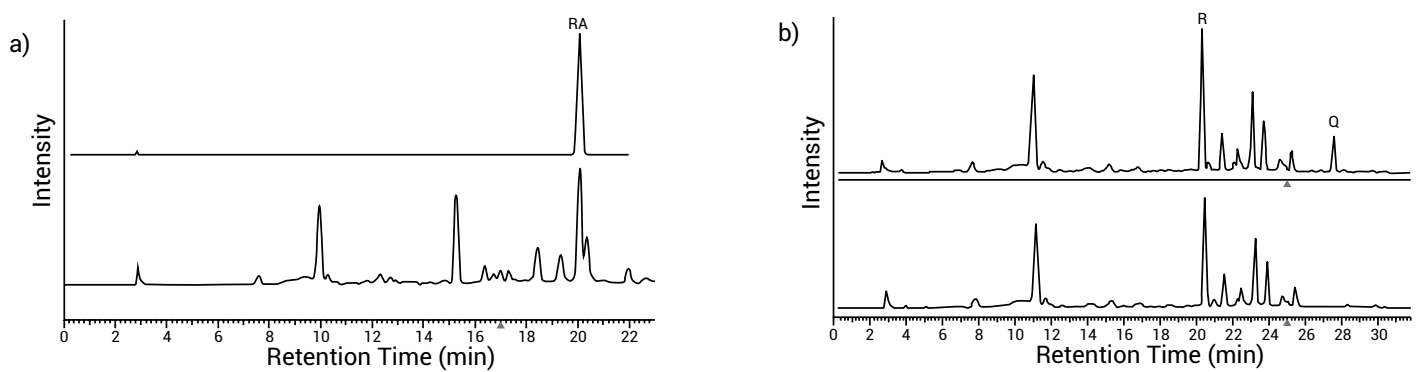

Figure 1. Chromatograms of (a) rosemary extract with rosmarinic acid (RA) standard (above), RT $20.2 \mathrm{~min}, 330 \mathrm{~nm}$, and (b) elderflower extract and spiked with rutin (R) and quercetin (Q) standards (above), rutin RT $20.5 \mathrm{~min}$, quercetin RT $28.5 \mathrm{~min}, 330 \mathrm{~nm}$. 
Table 2. The composition of hydrogels with the tested glycolic extract.

\begin{tabular}{|c|c|c|}
\hline The composition of hydrogel & Contents $(\mathrm{g})$ & Function in the formulation \\
\hline Aqua & 38.75 & Solvent \\
\hline Carbopol $^{\circledR}$ Ultrez 10 Polymer & 0.5 & Polymer, viscosity controlling agent \\
\hline $\begin{array}{c}\text { Triethanolamine } \\
\text { R. officinalis extract/ } \\
\text { S. nigra } \text { extract }\end{array}$ & 0.75 & $\begin{array}{c}\mathrm{pH} \text { regulator, alkalizing agent, supports the } \\
\text { gelling process }\end{array}$ \\
\hline Ethyl alcohol & 6.0 & Bioactive components \\
\hline & 4.0 & $\begin{array}{c}\text { Solvent, preservative, increases the absorption of } \\
\text { active ingredients }\end{array}$ \\
\hline
\end{tabular}

showed low values of $\%$ RSD $(<2)$ for inter-day variation. There was no significant change observed for the chromatograms of the standard solution and the experimental solution.

\section{Evaluation of antioxidant activity}

The antioxidant properties of the extracts of R. officinalis L. and S. nigra L. were determined using DPPH (2.2-diphenyl-1-picrylhydrazyl) radical assay, measured by the electron paramagnetic resonance (EPR) spectroscopy with microwaves of frequency of $9.3 \mathrm{GHz}$ from an X-band. EPR spectra of DPPH were recorded on a MiniScope MS 200 EPR spectrometer (Magnettech GmbH, Germany), under the following conditions: center field $330.48 \mathrm{mT}$, range of sweep: $9.92 \mathrm{mT}$, amplitude of modulation: $0.10 \mathrm{mT}$, microwave power: $12 \mathrm{~mW}$, sweep time: $20 \mathrm{~s}$, room temperature: at $25^{\circ} \mathrm{C}$. DPPH solution plus glycol was used as a negative control, and glycolic extracts of rosemary or glycolic extract of elderflower were used as a positive control. Briefly, $300 \mu \mathrm{L}$ of a rosemary extract solution and elderflower extract (in methanol, $0.5 \mathrm{mg} / \mathrm{mL}$ ) or glycol (as a blank) was mixed with $1200 \mu \mathrm{L}$ and $800 \mu \mathrm{L}$, respectively of a $1.3 \mathrm{mM}$ DPPH solution and held for 20 minutes in the dark. Then the residual DPPH content was determined by EPR spectroscopy. The results were presented as the amount of mg of the DPPH radical that was neutralized by $1 \mathrm{~mL}$ of the extract. The EPR spectra were recorded three for each sample. Data used for calculations were presented as the mean of three measurements (double integral of the signal) for three samples of each extract.

\section{Preparation of hydrogel formulations}

Hydrogels based on carbomer polymer containing the tested extracts were prepared to examine pharmaceutical availability using standard procedures described by Szcześniak and Pluta (22). The composition of formulations is shown in Table 2. The carbomer was slowly dispersed in water, mixed, homogenized, and left for swelling for 1 hour. Next, the neutralization was performed by adding the triethanolamine. The other components were then added. The formulations were stored in a fridge for one day prior to measurements.

The $\mathrm{pH}$ values of the prepared hydrogel formulations, determined using a $\mathrm{pH}$ meter CP-501 with EPS-1 electrode (Elmetron, Poland), were within limits for human skin: for hydrogel with rosemary extract $-\mathrm{pH}$ 6.65, with elderflower extract $-\mathrm{pH} 7.48$. The $\mathrm{pH}$ values of the obtained hydrogels were within the recommended limits for cosmetic and medicinal preparations applied to the skin (23).

\section{Release study of active compounds}

The studies on the pharmaceutical availability of glycolic extracts of rosemary and elderflower from the prepared hydrogels were performed under in vitro conditions using a simplified model of human skin represented by a semipermeable, cellulose dialysis membrane (dialysis membrane MembraCel ${ }^{\mathrm{TM}}, 44$ mm, 30 m, Roth, Karlsruhe, Germany). The acceptor fluid was a phosphate buffer $\mathrm{pH} 5.8$ (with acidity closer to the natural $\mathrm{pH}$ of the skin than PBS buffer $\mathrm{pH} 7.2$ that is more frequently used). The membrane with $4 \mathrm{~g}$ of hydrogel formulation was immersed vertically in a double-walled beaker containing $100 \mathrm{~mL}$ of phosphate buffer, thermostated by the water jacket, and mixed using a magnetic stirrer. The release studies were carried out in vitro at $32^{\circ} \mathrm{C}$, recommended by Polish Pharmacopoeia $\mathrm{X}$ (24) to study transdermal therapeutic systems. The speed of the release process was determined by measuring the quantity of the substances released at defined time intervals, using quercetin as a reference. The samples of $2 \mathrm{~mL}$ were taken after $0.5 \mathrm{~h}, 1 \mathrm{~h}, 2 \mathrm{~h}$, $3 \mathrm{~h}, 4 \mathrm{~h}$, and $5 \mathrm{~h}$, replaced with an equal volume of phosphate buffer to a volume of $100 \mathrm{~mL}$. Each sample was prepared in triplicate. The released polyphenols content was determined spectrophotometrically by the use of Evolution 60S UV-Vis spectrophotometer (Thermo Scientific, USA) and expressed as the quercetin equivalent in a sample, according to the 
standard curve equation: $\mathrm{Abs}=26.6730 \mathrm{x}-0.0058$ $\left(\mathrm{R}^{2}=0.995\right)$, where Abs - absorbance at $325 \mathrm{~nm}$, $\mathrm{x}$ - concentration of quercetin $(\mathrm{mg} / \mathrm{mL}$ ) (quercetin concentration range $0.0008-0.016 \mathrm{mg} / \mathrm{mL}$ ). The amount of substance released after time $t(\mathrm{~min})$ was calculated according to Hudemowicz et al. (25), followed the equation:

$$
M_{t}=\frac{V}{v} m_{t}+\sum m_{i}
$$

where:

$\mathrm{M}_{\mathrm{t}}$ - the amount of substance released over time $\mathrm{t}$,

$\mathrm{V}$ - the volume of fluid ( $\mathrm{mL}$ ) inside the apparatus,

$\mathrm{v}$ - the volume of fluid $(\mathrm{mL})$ taken once from the apparatus for dialysis,

$m_{t}$ - the amount of substance found in the sample,

$\mathrm{m}_{\mathrm{i}}$ - amount of substance found in samples v previously taken,

$\Sigma \mathrm{m}_{\mathrm{i}}$ - amount of active substance (sum) determined in the samples previously taken.

\section{Statistical analysis}

Analysis of all parameters in the extracts samples was performed in triplicate. Statistica 9.0 StatSoft was used to analyze the data. One-way ANOVA, with a Tukey's test, was applied to evaluate the significant differences among means at a level of $\mathrm{p}<0.05$.

\section{RESULTS AND DISCUSSION}

There is a renewed interest in components of natural origin. Plant extracts are mixtures composed of many constituents, displaying a very wide spectrum of chemical and biological activity. One of the most important active compounds of plant origin are polyphenols. Rosemary leaves and elderflowers are examples of herbal raw materials that have long been known and used in medicine, as well as in the pharmaceutical and cosmetics industry. Both are valued due to the content of biologically active compounds, including polyphenolic compounds. The results of the total content of polyphenols (TPC) and flavonoids (TFC) determination in glycolic extracts of rosemary and elderflower are compared in Table 3. The results of the performed tests showed that the glycolic rosemary extract is a richer source of these compounds than glycolic elderflower extract.

It was reported that the origin of the raw material and the solvent used in extraction may be important in the phenolic content. In a study by Afonso et al. (26), the content of total polyphenols in aqueous extracts calculated as gallic acid was $16.67 \mathrm{mg} / \mathrm{g}$. Research by Erkan et al. (27) indicates that the total phenolic content of ethanolic rosemary extract was $162 \mathrm{mg} \mathrm{GAE} / \mathrm{g}$. In reference to elderflowers, the results of literature research indicate a higher content of phenolic compounds in the examined material than data from our study. As reported by Stoilova et al. (28), the total phenolic content of elderflower extract was $194.0 \mathrm{mg}$ GAE/g dry extract. In studies conducted by Viapiana and Wesołowski (11) the total polyphenol content in black elder infusion was within the limits of 15.23-35.57 mg GAE/g depending on the origin of the raw material.

It can also be observed that the flavonoids predominate among the polyphenols in the studied extracts. The results of our research showed that the glycolic extract of rosemary exceeds the glycolic extract of elderflower also in terms of the flavonoid content. With reference to rosemary extract, they account for $92 \%$, while in the elderflower extract studied the contribution of flavonoids to the total polyphenol pool is $80.4 \%$. Therefore, both rosemary and elderflower can be classified as flavonoid raw materials. Studies aimed at comparing the polyphenol profile of various rosemary extracts obtained from fresh and dried raw materials indicate a high content of flavonoids $(0.270 \mathrm{mg} / \mathrm{mL}$ luteolin $)$ in water-alcoholic extracts obtained from fresh raw material (29). In the case of testing the chemical composition of infusion from elderflowers, the flavonoid content was 5.27-13.19 mg/g dry matter (calculated as rutin) (11).

The samples received from the manufacturer for testing had only the percentage of extract indicated, without standardization. Therefore, HPLC analysis was performed to determine the content of ingredients typically listed in the extract characteristics.

Table 3. Total content of polyphenols, flavonoids, and the antioxidant activity of glycolic extracts of rosemary and elderflower.

\begin{tabular}{|c|c|c|c|}
\hline Glycolic extract & $\begin{array}{c}\text { TPC } \\
(\mathrm{mgGAE} / 100 \mathrm{~mL} \pm \mathrm{SD})\end{array}$ & $\begin{array}{c}\text { TFC } \\
(\mathrm{mgCE} / 100 \mathrm{~mL} \pm \mathrm{SD})\end{array}$ & $\begin{array}{c}\text { Scavenging ability of DPPH radical } \\
(\mathrm{mg} / \mathrm{mL} \pm \mathrm{SD})\end{array}$ \\
\hline Rosemary & $179^{\mathrm{a}} \pm 18$ & $165^{\mathrm{a}} \pm 4$ & $40.5^{\mathrm{a}} \pm 0.3$ \\
\hline Elderflower & $145^{\mathrm{b}} \pm 8$ & $117^{\mathrm{b}} \pm 6$ & $26.1^{\mathrm{b}} \pm 0.1$ \\
\hline
\end{tabular}

TPC - total polyphenols content, TFC - total flavonoids content, GAE - gallic acid equivalent, CE - catechin equivalent, SD - standard deviation, DPPH - 2.2-diphenyl-1-picrylhydrazyl, values followed by different letter are statistically significantly different $(\mathrm{p}<0.05)$ 
The HPLC analysis showed that rosmarinic acid $(32.7 \pm 0.3 \mathrm{mg} / 100 \mathrm{~mL})$ is one of the dominant components of rosemary extract, while elderflower extract contains a high amount of rutin $(81.3 \pm$ $0.3 \mathrm{mg} / 100 \mathrm{~mL}$ ).

Glycolic extracts of rosemary and elderflower differ in antioxidant activity, which is directly proportional to the polyphenols and flavonoids content (Table 3). The extract of rosemary exceeds almost twice the elderflower extract in terms of the scavenging ability of the DPPH radical. The close correlation between antioxidant activity and phenolic content of plant extracts has been demonstrated by many authors (11, 21, 26-28). Research by Stoilova et al. (28) indicates that $S$. nigra extract at $0.152 \mu \mathrm{g} /$ $\mathrm{mL}$ caused 50\% DPPH scavenging (IC50). Erkan et al. (27) found that $R$. officinalis extract caused 50\% inhibition at $54.0 \mu \mathrm{g} / \mathrm{mL}$ concentration.

Rosemary and elderflower, the raw materials rich in polyphenols with antioxidant properties, could be used as active ingredients in new formulations. The penetration of active substances deep into the skin depends not only on the active ingredient and skin condition but also on the chosen carrier. Although in the past no attention was paid to the properties of the base itself, over time it has been shown that the choice of the base depends on the release of the active substance from pharmaceutical and cosmetic preparations and its absorption through the skin. In the medical and pharmaceutical sectors, the hydrogel base occupies an important place. The advantages of hydrogel substrates include easy application, transparency, and cooling sensation after application on the skin, which is a beneficial effect in many skin diseases. In addition, they neither influence the active substance nor lead to sensitization. Due to the above, hydrogels are recommended in the case of intolerance to lipophilic substrates, as well as in wound treatment, acute inflammation of the skin,

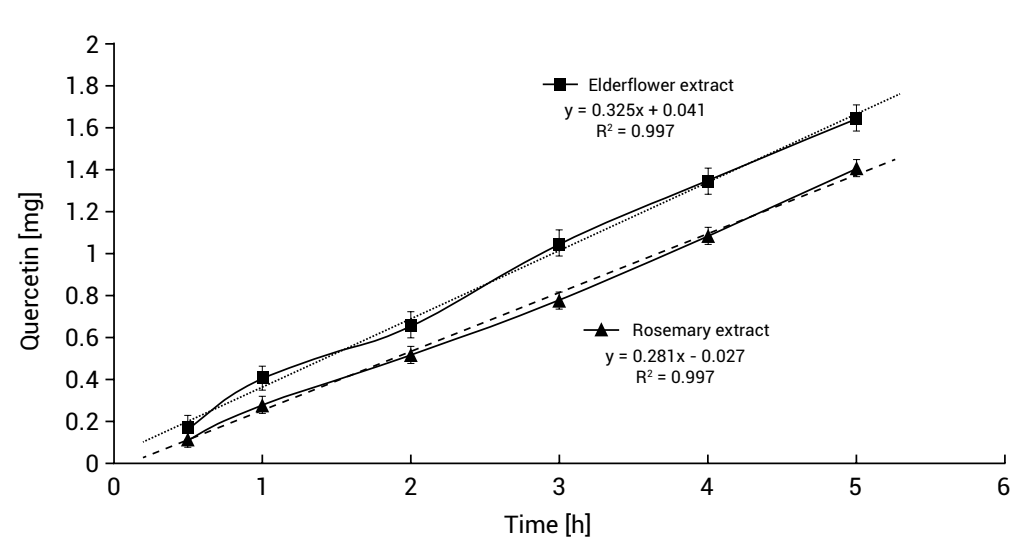

Figure 2. Zero-order kinetics of release of the active substance from hydrogels with rosemary and elderflower glycolic extracts. urticaria, sunburn, atopic dermatitis, or psoriasis. Moreover, hydrogels can be used as carriers of not only medicinal substances but also active ingredients with a care effect $(30,31)$.

The skin permeation ability of active substances appears to be very significant both for the researchers and for producers. The release test may concern both substances used therapeutically in dermatology, as well as prophylactically in cosmetology. Among them, salicylic acid (32), retinoic acid (33), vitamins (E, C, B, provitamin A) (34), and sunscreens (e.g. octylmethoxycinnamate (OMC) (32) can be mentioned. Only a few papers on the skin permeation of polyphenols $(35)$ and flavonoids $(36,37)$ exist. Based on the available literature, it can be concluded to state that the studies of pharmaceutical availability of plant extracts are rather limited. At the same time, research results indicate that new raw materials of plant origin are still being sought for the proper functioning and maintenance of the skin in good condition. Undoubtedly, biologically active plant substances perform important functions in dermatology and cosmetology. However, there is a need to conduct research to confirm their composition and properties in the hope of increasing the general knowledge about the effects of preparations of natural origin, in the recipe of which the ingredient or plant ingredients are contained. The biological activity of cosmetics enriched with plant polyphenols, including the antioxidant effect, is conditioned by the ability of their penetration through the skin barrier. In the case of topical preparations, it is important to release the active substance from the cosmetic form, the ability to reach the skin and eventually overcome the stratum corneum and penetrate deep into the epidermis and the dermis (9). The in vitro release tests of the active substance confirmed the usefulness of this method to assess the effectiveness of semisolid formulations. An important element of the action of active substances is their solubility and the ability to cross the barrier of the epidermis into deeper layers of the skin where they can interact. Thanks to the use of the dialysis membrane, which was a model system in relation to the human skin, the ability to pass the tested glycolic plant extracts from $R$. officinalis and $S$. nigra through the first skin barrier was confirmed. The kinetics of the release from the prepared hydrogels to the 
acceptor fluid was calculated based on the quercetin content as the ratio between the amount of active substance released and the time of exposure (Figure 2).

The performed experiment allows one to evaluate the release rate of quercetin from the hydrogel as a carrier for the tested extract. There were no significant differences in the release kinetics of the two extracts. The release data were found to be best fitted to the zero-order model, with the correlation coefficients 0.997 for both extracts (Figure 2). The extracts contain mainly phenolic, low molecular weight compounds, so the mechanism of diffusion through the membrane can follow the Fickian diffusion model, as was observed by Belščak-Cvitanović et al. (38). The compounds can easily permeate through the carbomer membrane without any interaction with polyacrylic acid polymers.

The permeation of active ingredients of plant origin into the skin is recognized as a very complex process, dependent on many different biochemical and physical factors. The most reliable data can be obtained during laboratory research provided in vivo as well as in vitro. The results of the pharmaceutical availability test are important due to the possibility of introducing changes in the formulation of the preparation by observing the release profile of the active substance, before beginning costly and timeconsuming in vivo application tests (19). The subject of skin penetration ability of active substances from plant extracts is still topical and requires further scientific investigation.

\section{CONCLUSION}

The outcomes of the study indicate that the extracts of $R$. officinalis and S. nigra can be used as a flavonoid-containing plant material with antioxidant properties. Model hydrogel formulations with rosemary and elderflower may serve as a basis for developing new preparations used in the care or treatment of skin diseases. Studies on the kinetics of the active substance release from hydrogel formulations constitute a tool for assessing the effectiveness of semisolid products.

\section{CONFLICT OF INTERESTS}

The authors declare that they have no conflict of interest.

\section{REFERENCES}

1. Ribeiro A.S., Estanqueiro M., Oliveira M.B., Lobo J.M.S.: Cosmetics 2, 48 (2015).
2. Sander C.S., Chang H., Hamm F., Elsner P., Thiele J.J.: Int. J. Dermatol. 43, 326 (2004).

3. Ashok B.T., Ali R.: Exp. Gerontol. 34, 293 (1999).

4. Wolfle U., Seelinger G., Bauer G., Meinke M.C., Lademann J., Schempp C.M.: Skin Pharmacol. Physiol. 27, 316 (2014).

5. Rhie G., Shin M.H., Seo J.Y., Choi W.W., Cho K.H., Kim K.H., Pak K.C., Eun H.C., Chung J.H.: J. Invest. Dermatol. 117, 1212 (2001).

6. Anunciato T.P., da Rocha Filho P.A.: J. Cosmet. Dermatol. 11, 51 (2012).

7. Han X., Shen T., Lou H.: Int. J. Mol. Sci. 8, 950 (2007).

8. Nichols J.A., Katiyar S.K.: Arch. Dermatol. Res. 302, 71 (2010).

9. Zillich O.V., Schweiggert-Weisz U., Eisner P., Kerscher M.: Int. J. Cosmet. Sci. 37, 455 (2015).

10. Borrás-Linares I., Stojanović Z., Quirantes-Piné R., Arráez-Román D., Švarc-Gajić J., et al.: Int. J. Mol. Sci. 15, 20585 (2014).

11. Viapiana A., Wesolowski M.: Plant Foods Hum. Nutr. 72, 82 (2017).

12. Bai N., He K., Roller M., Lai C.S., Shao X., et al.: J. Agr. Food Chem. 58, 5363 (2010).

13. Mena P., Cirlini M., Tassotti M., Herrlinger K.A., Dall'Asta Ch., Del Rio D.: Molecules 21, 1576 (2016).

14. Al-Sereiti M.R., Abu-Amer K.M., Sen P.: Indian J. Exp. Biol. 37, 124 (1999).

15. Altinier G., Sosa S., Aquino R.P., Mencherini T., Della Loggia R., Tubaro A.: J. Agr. Food Chem. 55, 1718 (2007).

16. Jarzycka A., Lewinska A., Gancarz R., Wilk K.A.: J. Photochem. Photobiol. B 128, 50 (2013).

17. Mogoŝanu D.G., Popescu F.C., Busuioc C.J., Pop O., Pârvănescu H., et al.: Studia Universitatis Vasile Goldiş, Seria Ştiinţele Vieţii 21, 70 (2011).

18. Otto A., du Plessis J., Wiechers J.W.: Int. J. Cosmet. Sci. 31, 1 (2009).

19. Olejnik A., Goscianska J., Nowak I.: J. Pharm. Sci. 101, 4032 (2012).

20. Agbor G.A., Vinson J.A., Donnelly P.E.: Int. J. Food Sci. Nutr. Diet. 3, 147 (2014).

21. Kim D-O., Jeong S.W., Lee C.Y.: Food Chem. 81, 321 (2003).

22. Szcześniak M., Pluta J.: Polim. Med. 41, 27 (2001).

23. Piechota-Urbańska M., Berner-Strzelczyk A.: Polim. Med. 41, 17 (2011).

24. Polish Pharmacopoeia edition X. Institute for Drug Research \& Control, Warsaw, Poland 2014.

25. Hudemowicz W., Piotrowska I., Sieradzki E.: Ointment. Transdermal therapeutic systems. 
Exercises with drug form technology, Medical University of Warsaw, Warsaw 2014 (in Polish).

26. Afonso M.S., de O Silva A.M., Carvalho E.B., Rivelli D.P., Barros S.B., et al.: Nutr. Metab. 10, 19 (2013).

27. Erkan N., Ayranci G., Ayranci E.: Food Chem. 110, 76 (2008).

28. Stoilova I., Wilker M., Stoyanova A., Krastanov A., Stanchev V.: Herba Pol. 53, 45 (2007).

29. Olah N.K., Osser G., Campean R.F., Furtuna F.R., Benedec D., et al.: Pak. J. Pharm. Sci. 29, 2355 (2016).

30. Zagórska-Dziok M., Sobczak M.: Pharmaceutics 12, 396 (2020).

31. Peppas N.A., Bures P., Leobandung W., Ichikawa H.: Eur. J. Pharm. Biopharm. 50, 27 (2000).

32. Montenegro L., Paolino D., Puglisi G.: J. Cosmet. Sci. 55, 509 (2004).
33. Thakker K.D., Chern W.H.: Dissolution Technol. 10, 10 (2003).

34. Gabbanini S., Matera R., Beltramini C., Minghetti A., Valgimigli I.: J. Pharm. Biomed. Anal. 52, 461 (2010).

35. Zillich O.V., Schweiggert-Weisz U., Hasenkopf K., Eisner P., Kerscher M.: Int. J. Cosmet. Sci. 35, 491 (2013).

36. Merfort I., Heilmann J., Hagedorn-Leweke U., Lippold B.C.: Pharmazie 49, 509 (1994).

37. Arct J., Jarnicka A., Mojski M.: Euro Cosmetics 5, 29 (2000).

38. Belščak-Cvitanović A., Stojanović R., Manojlović V., Komes D., Cindrić I.J., et al.: Food Res. Int. 44, 1094 (2011).

(C) 2021 by Polish Pharmaceutical Society. This is an open-access article under the CC BY NC license (http://creativecommons.org/licenses/BY/4.0/). 